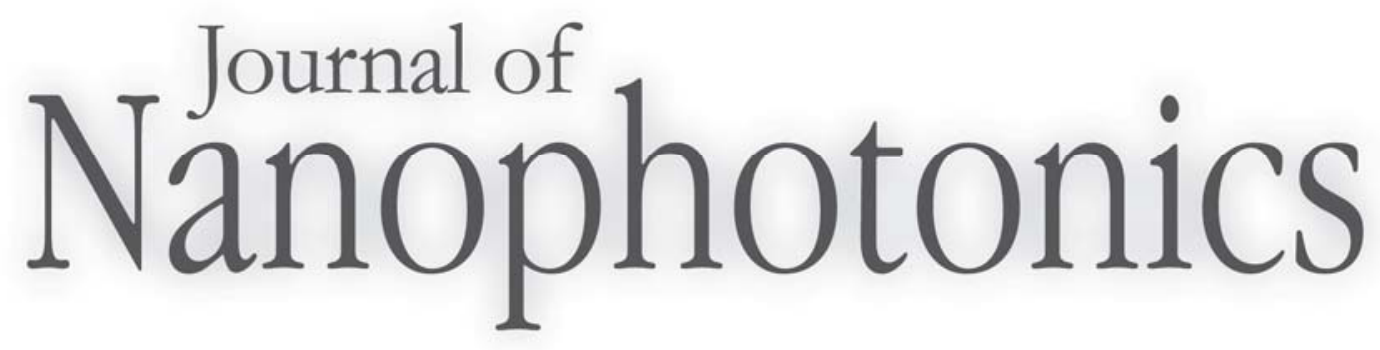

SPIEDigitalLibrary.org/jnp

\title{
Commentary: Multichannel ellipsometry for monitoring processes
}

Ilsin An 


\title{
COMMENTARY
}

\section{Multichannel ellipsometry for monitoring processes}

\author{
Ilsin An \\ Hanyang University, Department of Applied Physics, Ansan 426-791, Korea \\ ilsin@hanyang.ac.kr
}

The demand for tighter quality control of thin films continues to rise. As the feature size of transistors shrinks, the thickness of dielectric films reduces to a few nanometers or less and therefore needs to be controlled with monolayer-scale precision. For successful nanopatterning in deep ultraviolet (DUV) lithography, the thicknesses of photoresists and antireflection coatings must be accurate and their optical properties must be known precisely. Precise control of composition and thickness uniformity of films is also necessary for largearea samples such as displays and thin-film solar-cell panels.

One of the most powerful techniques for thin-film characterization is spectroscopic ellipsometry (SE). Since the introduction of laboratory automation, spectroscopic ellipsometry has increased in popularity as a non-destructive tool for characterizing the optical properties as well as the microstructure of thin films. Spectroscopic ellipsometry measures two parameters $\{\Delta, \Psi\}$ as functions of (free-space) wavelength. These parameters represent changes in polarization states upon reflection from the sample. SE has two remarkable advantages over other techniques for thin-film characterization: (i) it determines microstructural quantities such as volume fractions of physical constituents and thickness of multi-layers with monolayer sensitivity, and (ii) it measures the spectra of both refractive index and extinction coefficient (i.e., absorption) simultaneously. These optical spectra can provide very useful information such as band structure and crystallinity of materials.

For decades, a prism or grating in a monochromator was rotated in order to scan the wavelength; it took tens of minutes to acquire $\sim 100$ spectral points with a single-channel detector. However, after the introduction of commercial photodiode arrays (PDAs) in the mid1980, fast spectral acquisition became possible [1]. The PDA has good spectral response from near-infrared to near-ultraviolet wavelengths and its 1024 channels can be scanned in few milliseconds. Thus, by adopting the combination of a PDA and a spectrograph as a spectroscopic detection system, real-time spectroscopic ellipsometry (RTSE) was realized for operation from 1.5 to $5.0 \mathrm{eV}$ in less than $100 \mathrm{~ms}$ [2].

RTSE has been applied successfully to monitor thin-film growth and surface modification. It reveals the temporal evolution of the optical properties and the layered structure of materials during growth and processing [3,4]. The typical configuration of a rotating polarizer type multichannel ellipsometry used for real-time measurement is shown in Fig. 1 (top left). It consists of (1) a broadband source, (2) a rotating polarizer, (3) optical windows, (4) a processing chamber, (5) an analyzer, and (6) a multichannel detector with spectrograph. Instead of a vacuum chamber, a chemical cell also can be used for monitoring of chemical processes in liquids (bottom left in Fig. 1).

As abundant results of RTSE on vacuum processes are available in literature, let us focus here on chemical processes. The right side of Fig. 1 shows the $\Delta$-spectrum collected during the photochemical reaction of a 7-nm-thick gold film in a chlorine-containing liquid cell. Ultraviolet radiation is provided from the behind of gold film using a mercury lamp. As the gold film is extremely thin and was deposited on fused silica glass, ultraviolet rays can penetrate the surface of the gold film and activate the formation of gold chloride which eventually desorbs from the surface. After 100 minutes of ultraviolot exposure, the $\Delta$ - 
spectrum becomes flat at zero, which is the indication of complete chlorination and removal of the film. Although this spectrum was accidentally recorded, the discovery of the chlorination of gold film without multichannel SE would not have been possible, because the etch rate $(\sim 0.001 \mathrm{~nm} / \mathrm{s})$ was too small to be detected with any other technique [5]. Besides, further analysis of $\{\Delta, \Psi\}$ spectra elucidated the evolution of the optical properties of gold caused by the reduction of grain size.
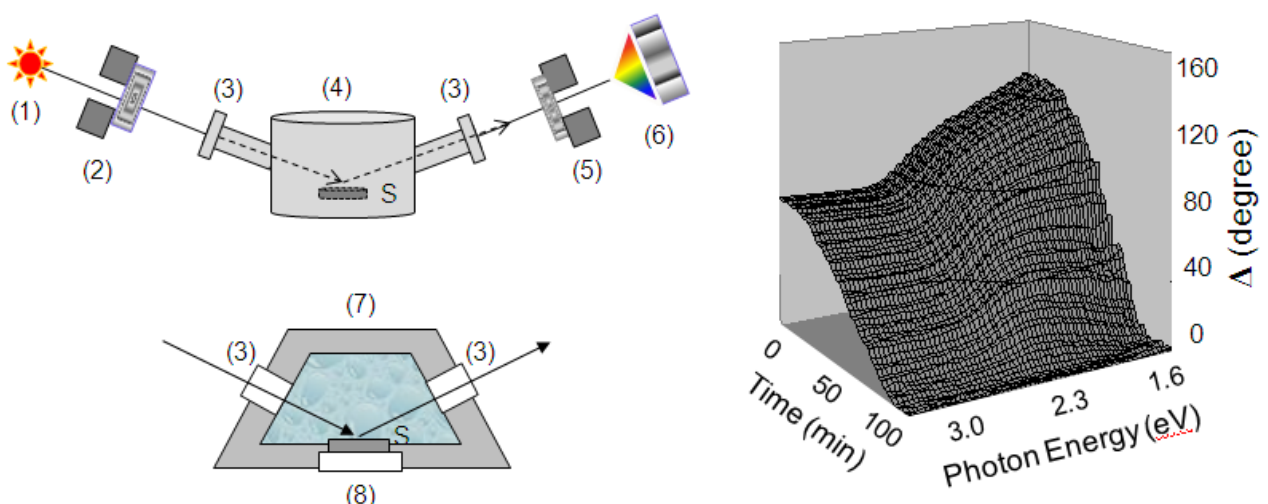

Fig. 1. Top left: Schematic of a multichannel ellipsometer integrated into vacuum chamber in which sample (S) is located. Bottom left: Top view of a chemical cell designed for photochemical processes. The cell (7) made of Kel-F is filled with a liquid and the gold thin film (S) can be illuminated by ultraviolet rays from the substrate (8). Right: $\Delta$-spectrum collected over $100 \mathrm{~min}$.

Nowadays, multichannel detectors can cover much wider spectral ranges, thanks to advances in semiconductor device technology. A multichannel detector made of an array of charge-coupled devices (CCDs) shows much higher sensitivity than a PDA ( 100 times) in the visible regime, and it can cover the near-ultraviolet regime when the surface of the CCD array is coated with a phosphor. Back-thinned CCD arrays cover much deeper into the vacuum ultraviolet (VUV) range due to increased quantum efficiency.

The recently developed VUV multichannel ellipsometry can be used to monitor the growth or annealing process of high-k and low-k materials which exhibit wide bandgap [6]. Also, it can be used to monitor DUV lithography processes. Instead of silicon, InGaAs is used in multichannel detectors which can cover near-infrared regime (900-2500 nm wavelengths). Near-infrared multichannel ellipsometry can be used to study small-bandgap materials such as CIGS films for solar cells. Currently, the spectral range of multichannel detectors can range from the near-infrared to the X-ray regimes. Also, the number of channels has increased and one-inch detectors with 4096 channels are commercially available for spectroscopy. With a properly chosen grating, the spectrum can be acquired with extremely high resolution. Furthermore, the scanning time of multichannel detectors has been reduced to the microsecond range for faster kinetic studies.

Another application of high-speed multichannel spectroscopic ellipsometry is mapping of large-area samples such as LCD panels and solar-cell panels for inspecting the uniformity of thickness or optical properties. Figure 2 (left) shows the retardance obtained from a LCD panel after rubbing a polyimide film. The rubbed surface provides the alignment anchor for the liquid crystal. The rubbing process induces surface anisotropy and thus, in this case a normal-incidence multichannel spectroscopic ellipsometer is used to detect the strength of rubbing process in terms of retardance. Retardance is highly sensitive to the rubbing 
parameters such as the kind of rubbing cloth, rubbing depth, and rubbing speed. The data in Fig. 2 (left) were obtained by increasing the number of rubbing strokes. These data show that the rubbing strength increased with the number of strokes but was reduced by excessive rubbing due to the gradual removal the polyimide layer. Thus, in order to produce a large display panel with uniform picture quality, the uniformity of rubbing strength along with other parameters needs to be checked.
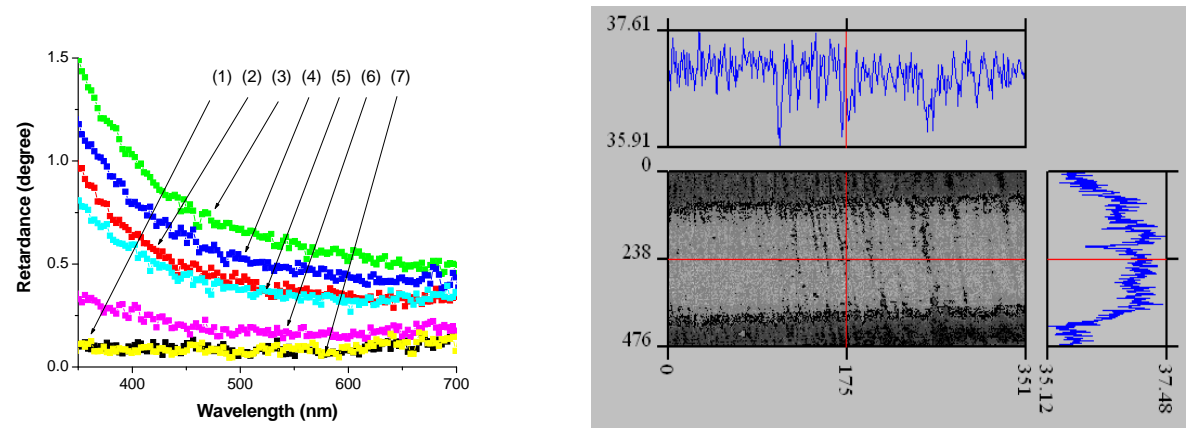

Fig. 2. Left: Retardance measured over a polyimide layer after rubbing. The number of rubbing strokes increased by unity from spectrum (1) to (7). Right: Image of $\Delta$ measured over a small area $(1.76 \times 2.38 \mathrm{~mm})$. Cross-sectional values of $\Delta$ are also shown in insets. Scales are pixel number with $5 \mu \mathrm{m} /$ pixel resolution. A singlewavelength diode $(530 \mathrm{~nm})$ was used as the light source.

Often, the uniformity over a small area can provide important information about a process. However, due to the beam size in a conventional ellipsometer, the spatial resolution of ellipsometry is limited to few $\mathrm{mm}$ in general. In this case, a two-dimensional multichannel detector and imaging optics can be employed to develop imaging ellipsometry, with which two-dimensional microscopic images of $\{\Delta, \Psi\}$ can be obtained in a few seconds. Although imaging ellipsometry is more popular for label-free biodetection [7], many useful applications are also found in the areas of materials processing and device fabrication. Figure 2 (right) shows $\Delta$-image collected over amorphous silicon (a-Si) after crystallization by $\mathrm{XeCl}$ laser annealing ( $351 \times 476$ pixels). The dark area is amorphous silicon and the white area indicates crystallization due to the passage of the laser beam. Without much analysis, details of the processing quality can be gleaned from the image itself. Imaging ellipsometry has also shown good sensitivity for detecting metal-induced crystallization of a-Si [8].

Imaging ellipsometry is a single wavelength ellipsometry in nature, as the image shown in Fig. 2 (right) is in the gray scale. However, by changing the wavelength, spectroscopic imaging ellipsometry can be implemented. In this case, more quantitative information can be deduced for each image point such as the thickness of a multilayer or the degree of crystallization. Moreover, two-dimensional arrays can be used to collect one-dimensional images along with the spectroscopic-ellipsometry spectrum in the other dimension for each image point [9].

In summary, the incorporation of high-speed spectral scanning in multichannel ellipsometry has opened up new application fields of real-time monitoring of thin-film processing and quality control of large-area samples. By expanding the spectral range and increasing the data-acquisition speed, multichannel ellipsometry will be applied to many different thin-film processes. In addition, two-dimensional multichannel detectors have brought micrometer-scale spatial resolution into ellipsometry. With increased resolution and by employing imaging optics for an extended spectral range, imaging ellipsometry will become a very powerful surface characterization technique with monolayer sensitivity. 


\section{Acknowledgments}

This work was supported by Consortium of Semiconductor Advanced Research (2007-2009).

\section{References}

[1] K. Vedam, "Spectroscopic ellipsometry: a historical overview," Thin Solid Films 313/314, 1-9 (1998) [doi:10.1016/S0040-6090(97)00762-1].

[2] R. W. Collins, I. An, H. Fujiwara, J. Lee, Y. Lu, J. Koh, and P. I. Rovira, "Advances in multichannel spectroscopic ellipsometry," Thin Solid Films 313-314, 18-32 (1998) [doi:10.1016/S0040-6090(97)00764-5].

[3] I. An, H. V. Nguyen, N. V. Nguyen, and R. W. Collins, "Microstructural evolution of ultrathin amorphous silicon films by real-time spectroscopic ellipsometry," Phys. Rev. Lett. 65, 2274-2277 (1990) [doi:10.1103/PhysRevLett.65.2274].

[4] C. Pickering, "Complementary in-situ and post-deposition diagnostics of thin film semiconductor structures," Thin Solid Films 313/314, 406-415 (1998) [doi:10.1016/S0040-6090(97)00855-9].

[5] I. An and D. Seong, "Morphological development and etching of gold thin film under UV-exposure in chlorine-based liquids," Chem. Lett. 33, 1232-1235 (2004) [doi:10.1246/cl.2004.1232].

[6] I. An, "Development of vacuum ultraviolet multichannel ellipsometry and its application to the characterization of ultrathin zirconium oxide films," $J$. Nanophoton. 2, 021905 (2008) [doi:10.1117/1.3025960].

[7] Z. Wang and G. Jin, "Covalent immobilization of proteins for the biosensor based on imaging ellipsometry," J. Immunol. Methods 285, 237-243 (2004) [doi:10.1016/j.jim.2003.12.002].

[8] A. J. Choi, T. H. Ghong, Y. D. Kim, J. H. Oh, and J. Jang, "Imaging ellipsometry study on the Ni-mediated crystallization of a-Si," J. Appl. Phys. 100, 113529 (2006) [doi:10.1063/1.2345468].

[9] W. Chegal, S. Kim, Y. Kwak, H. Cho, and Y Lee, "Mono-axial power spectrograph for a spectral imaging ellipsometer: design and experimental results," Meas. Sci. Technol. 14, 558-562 (2003) [doi:10.1088/0957-0233/14/5/304]. 